\title{
Electrophoretic hydrogel adhesion for fabrication of three-dimensional materials
}

\author{
Taka-Aki Asoh ${ }^{1,2}$ \\ The adhesion of hydrogels has attracted substantial attention for use in building three-dimensional constructs such as scaffolds \\ for cell culture, carriers of drug release system and soft actuators. This review article summarizes our group's research on \\ electrophoretic hydrogel adhesion, a novel strategy for the adhesion of hydrogels. This technique involves the electrophoretic \\ manipulation of a polyelectrolyte to form an adhesive layer at the interface of the two hydrogels and is a powerful method for \\ fabrication of free-form hydrogel constructs through the adhesion of microgels.
}

Polymer Journal (2016) 48, 1095-1101; doi:10.1038/pj.2016.85; published online 21 September 2016

\section{INTRODUCTION}

Hydrogels are fascinating materials because they are water-swollen, similarly to living soft tissue. Therefore, researchers have reported many applications of hydrogels, including use as scaffolds for cell culture, ${ }^{1}$ drug delivery carriers ${ }^{2}$ and soft actuators. ${ }^{3-6}$ The fabrication of highly organized three-dimensional (3D) soft materials, such as living tissue, has attracted substantial attention, and 3D architectures consisting of biocompatible polymers, proteins and cells have been prepared. $^{7-9}$

$3 \mathrm{D}$-printing technologies have recently received substantial attention because they allow precise control of both structure and material properties in a customizable fashion. Generally, the additive manufacturing (AM) of polymeric devises is based on fused deposition modeling of thermoplastic polymers or a chemical reaction of photoreactive monomers and/or macromonomers. ${ }^{10}$ Although soft tissues have been prepared by direct writing with a hydrogel precursor ink in a support bath, such as a thermoresponsive so-gel solution or a hydrogel reservoir, ${ }^{11-13}$ precursors of hydrogels are often a onecomponent solution, and AM technology is limited in its ability to 3D print a highly organized hydrogel architecture consisting of multiple components.

Because the adhesion of materials is one of the most important and simplest processes for building $3 \mathrm{D}$ architecture, the adhesion of hydrogels is required to develop functional devices using biocompatible or stimuli-responsive hydrogels. However, the usual glues for the adhesion of hard materials cannot be used to adhere hydrogels because the presence of solidified glues at the adhesive interface limits the softness and/or mass transfer between two adhered hydrogels.

Materials are designed to adhere to each other only through interactions involving the outermost surface. Various interactions, such as metal-ligand, ${ }^{14}$ electrostatic, ${ }^{15-18}$ host-guest ${ }^{19}$ and organicinorganic $^{20-23}$ interactions, have also been used for the adhesion of soft materials, including hydrogels. However, spontaneous adhesion is disadvantageous for the control of the physical properties of obtained materials, such as configuration, shape, mechanical strength and elasticity. Therefore, a novel method for the adhesion of hydrogels is required for the rapid and simple fabrication of highly organized hydrogel architectures.

In this review article, I provide an overview of the research on a novel strategy for the adhesion of hydrogels, focusing on the electrophoretic adhesion of hydrogels ${ }^{24-29}$ performed by my research group. The electrophoretic manipulation of a polyelectrolyte has been used for the adhesion of hydrogels (Figure 1). This new technique has attracted substantial attention for its potential in the development of on-demand fabrication of free-form hydrogel architectures that can function in water.

\section{DIRECT-CURRENT ELECTROPHORETIC ADHESION OF OPPOSITELY CHARGED HYDROGELS}

The rapid adhesion of cationic and anionic hydrogels was achieved by applying a direct-current (DC) electric field between two hydrogels. ${ }^{24}$ First, we adhered the naturally occurring polymer gels. The chemical structures of the polyelectrolytes used in this experiment are shown in Figure 2a. The hydrogels were prepared by the chemical crosslinking of the amines and carboxylic acids of cationic gelatin type A. The cationic and anionic gels were prepared by the addition of chitosan hydrochloride or sodium poly( $\gamma$-glutamate) (PGA) at $10 \%$ of the total polymer concentration to the gelatin solution to prepare chitosan 10 and PGA10 gels, respectively, and were used for the adhesion experiment. The chitosan 10 gel did not adhere to the PGA10 gel through contact alone. However, the two hydrogels adhered to each other when a DC electric field was applied with a cationic gel on the anode and an anionic gel on the cathode (Figures 1 and $2 \mathrm{~b}$ ). The adhesion of the two hydrogels was not observed in the case of applying the opposite electric field. As shown in Figure 2c, the adhesion

\footnotetext{
${ }^{1}$ Advanced Research Institute for Natural Science and Technology, Osaka City University, Osaka, Japan and ${ }^{2}$ Department of Chemistry, Osaka City University, Osaka, Japan Correspondence: Dr T Asoh, Advanced Research Institute for Natural Science and Technology, Osaka City University, 3-3-138 Sugimoto, Sumiyoshi-ku, Osaka 558-8585, Japan. E-mail: asoh@sci.osaka-cu.ac.jp

Received 15 March 2016; revised 31 July 2016; accepted 1 August 2016; published online 21 September 2016
} 
interface of the hydrogels withstood the torsional stress, and the adhesive interface was stable without detachment by immersion in deionized water. It is well known that negatively and positively charged polymers move to the anode and cathode during electrophoresis, respectively. Then, water-insoluble polyion complexes (PICs) as an adhesive layer were formed at the interface of the two hydrogels (Figure 1). To measure the adhesive strength, a tensile test of the adhered gels was carried out under ambient atmosphere. It took only

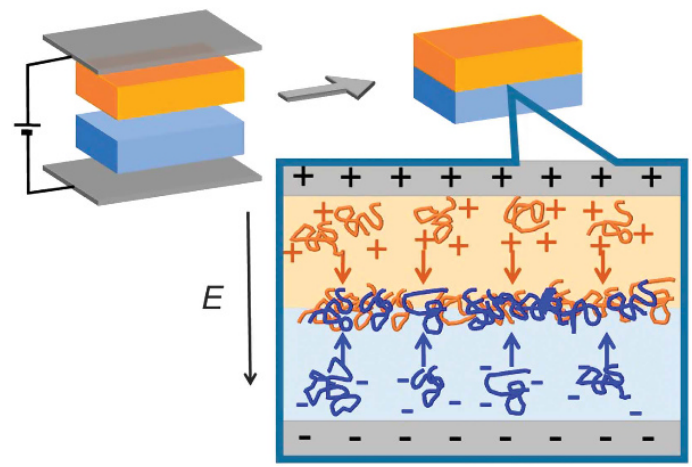

Figure 1 Schematic illustration of the direct-current electrophoretic adhesion of cationic and anionic hydrogels. Cationic and anionic polymers are shown in orange and blue, respectively. 10-20s to achieve the electrophoretic adhesion when the applied voltage was $2.5 \mathrm{~V} \mathrm{~mm}^{-1}$. The adhesive strength increased with increasing electrophoresis time, and the chitosan10/PGA10 gels showed stronger adhesion than the gelatin/PGA10 gels (Figure 2d). When the concentration of PGA increased to $20 \%$, the adhesive strength of the chitosan10/PGA20 gels was stronger than the hydrogels themselves, and the adhesive strength was not measured because the chitosan10 or PGA20 gels broke without detachment during the tensile test. These results indicate that the driving force of adhesion of these hydrogels is electrostatic interaction. Cationic and anionic polymers are concentrated at the interface of the two hydrogels by electrophoresis, and then form a PIC layer, causing the hydrogels to adhere. The detachment of the adhered gels was observed by applying an inverse electric field. During the inverse electrophoresis, the adhesive strength decreased with increasing electrophoresis time, as shown in Figure 2e. Eventually, the hydrogels detached within $10 \mathrm{~s}$ because the polymers moved in opposite directions, and then the PIC as an adhesive layer was deconstructed by the inverse electrophoresis. The electrophoretic adhesion and detachment of the hydrogels were repeated for multiple cycles. The adhesive strength of the adhesion and detachment cycles is shown in Figure $2 \mathrm{f}$. The gels detached by an inverse electric field were re-adhered by applying an ordered inverse electric field. Even after 10 cycles of adhesion/detachment, the adhesive strength was still $75 \%$ of the initial value. ${ }^{25}$ a

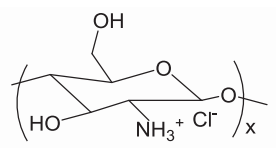

Chitosan<smiles>CC(C)(C)CCC(NC(C)(C)C)C(=O)O[Na]</smiles>

PGA

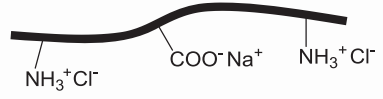

b

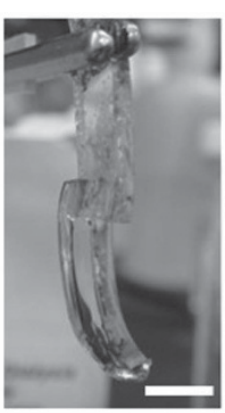

C

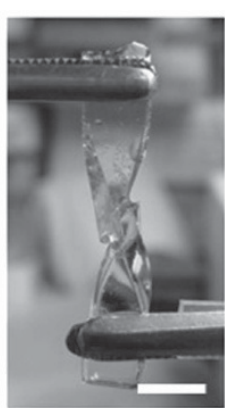

Gelatin
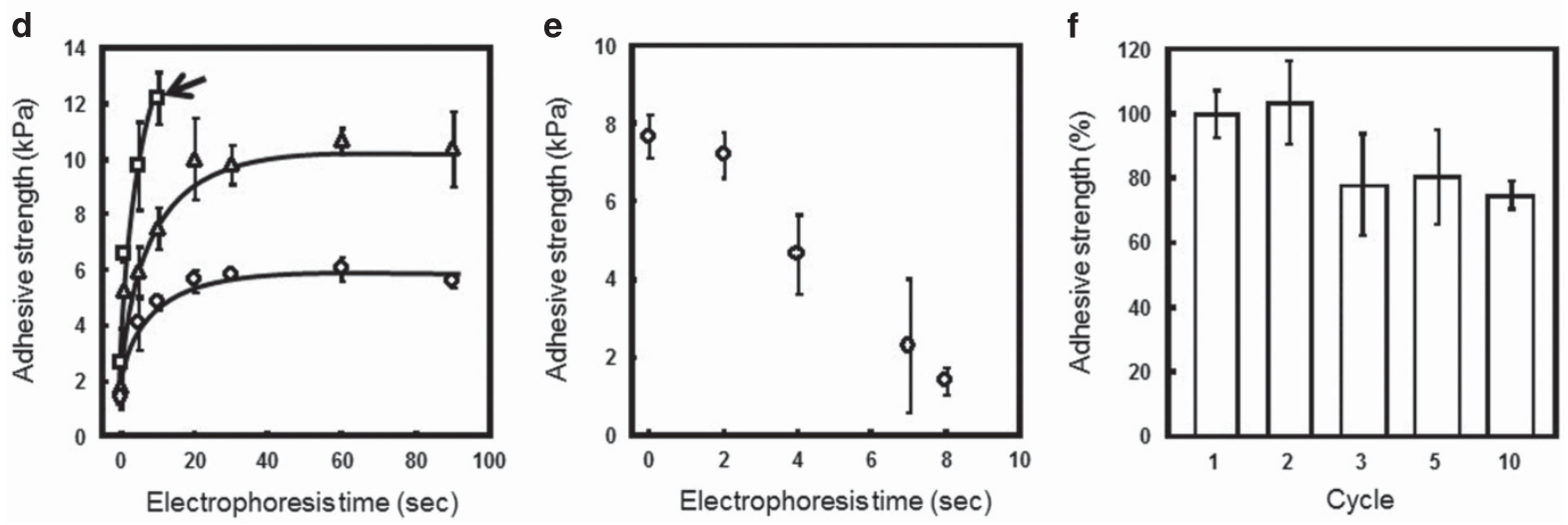

Figure 2 (a) Chemical structures of chitosan, poly $(\gamma$-glutamate) (PGA) and gelatin. Macroscopic images of (b) adhered and (c) twisted gels. The chitosan and PGA gels were stained with an orange and a blue dye. (d) The adhesive strengths of hydrogels as a function of the electrophoresis time $(n=3)$. Circle, triangle and square symbols indicate the adhered gelatin/PGA10, chitosan10/PGA10 and chitosan10/PGA20 gels, respectively. The arrow inside the figure indicates where the adhered gels broke before detachment. (e) The adhesive strengths of gelatin/PGA10 gels during detachment process $(n=3$ ). (f) The adhesive strengths during repeated adhesion and detachment cycles $(n=3)$. The hydrogels were adhered by an ordered electrophoresis, and the adhered gels were then detached by an inverse electrophoresis. All scale bars are $5 \mathrm{~mm}$. Reproduced from Asoh et al. ${ }^{25}$ from the Royal Society of Chemistry. A full color version of this figure is available at Polymer Journal online. 


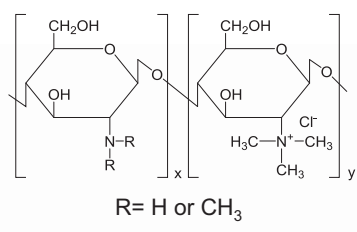

TMC

b

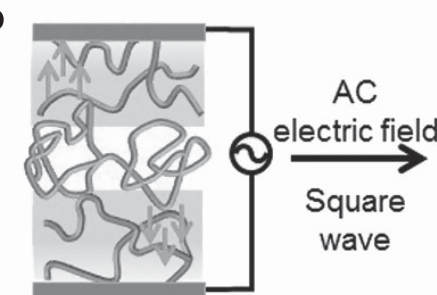

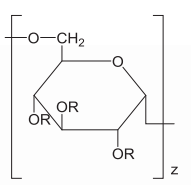

$\mathrm{R}=\mathrm{H}$ or $\mathrm{SO}_{3}{ }^{-} \mathrm{Na}^{+}$

DS

C

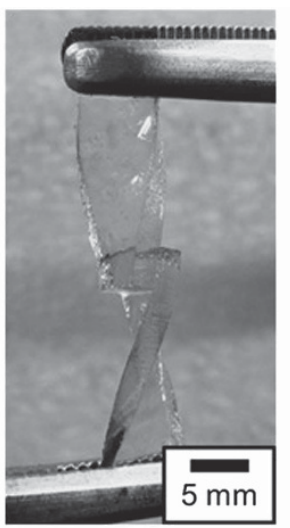

d

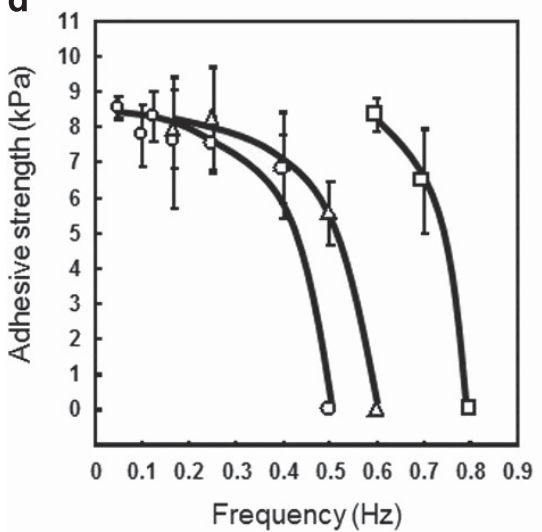

e

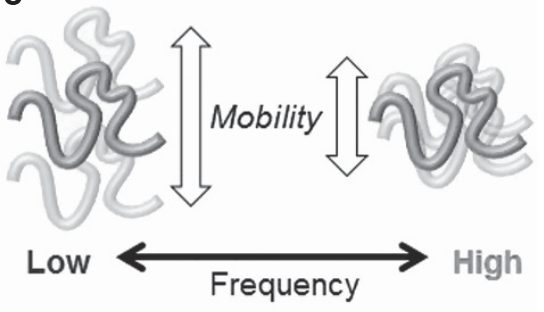

Frequency $(\mathrm{Hz})$

Figure 3 (a) Chemical structures of TMC and dextran sulfate (DS). (b) An illustration of the experimental procedure for the alternating-current electrophoretic adhesion of similarly charged DS gels. The cationic TMC polymers and anionic DS networks are shown in orange and blue, respectively. (c) Macroscopic images of the adhered DS gels. The DS gels were stained with a blue dye. (d) The adhesive strengths of the DS gels as a function of the frequency when a square wave was applied $(n=5)$. Circle, triangle and square symbols indicate 5,10 and 20 periods, respectively. (e) The oscillation mobility of TMC at the gel interface when the frequency was changed. Reproduced from Asoh et al. ${ }^{26}$ from Elsevier. A full color version of this figure is available at Polymer Journal online.

\section{ALTERNATING-CURRENT ELECTROPHORETIC ADHESION OF LIKE-CHARGED OR NON-IONIC HYDROGELS}

In a study of the electrophoretic adhesion of hydrogels using a weak polyelectrolyte (for example, a primary amine salt and a carboxylate) and a DC electric field, the detachment of the adhered gels was obtained by an inverse electric field. ${ }^{25}$ Although reversible adhesion and detachment are promising, it is difficult to adhere similarly charged gels by DC electrophoretic adhesion. To adhere similarly charged gels, we used a combination of an alternatingcurrent (AC) electric field and a strong polyelectrolyte gel (Figure 3a). ${ }^{26}$ Anionic sodium dextran sulfate (DS) gels, which were prepared by the chemical crosslinking of gelatin type A with DS, and cationic quaternized chitosan $\mathrm{N}, \mathrm{N}, \mathrm{N}$-trimetyl chitosan chloride (TMC) were used for the adhesion experiment. When a droplet of the TMC aqueous solution was sandwiched between two DS gels, adhesion was not observed after pressure bonding and applying the DC electric field. However, two DS gels adhered after application of an AC electric field with a square wave (Figure 3b and c). The adhesive strength of the DS gels decreased with increasing frequency and increased with increasing periodicity (Figure 3d). The oscillations of the TMC at two DS gel interfaces by $\mathrm{AC}$ electrophoresis had a key role in the PIC formation and hydrogel adhesion. For constant electrophoresis periods, the mobilities of TMC were lower when the frequency was higher. In contrast, TMC was able to move a long distance at a lower frequency (Figure 3e). When the frequency was low $(0.1-0.4 \mathrm{~Hz})$, hydrogel adhesion was observed at early periods (Figure $3 \mathrm{~d}$; circle) because of the long-distance movement of TMC. However, when the frequency was high $(0.5-0.7 \mathrm{~Hz})$, the hydrogels adhered by increasing the periods to 10 and 20 (Figure $3 \mathrm{~d}$; triangle and square). These results indicate that the TMC oscillation at the gel interfaces has a key role in the AC electrophoretic adhesion of hydrogels. Through AC electrophoretic adhesion, the joining of non-ionic poly(vinyl alcohol) (PVA) gels was also possible. ${ }^{27}$ In this case, we used poly(3-methacrylamidophenylboronic acid-co$\mathrm{N}, \mathrm{N}$-dimethylacrylamide) (poly(MAPBA-co-DMAAm)) as a binder (Figure 4a). Phenylboronic acid (PBA) shows a reversible bonding with diols around their $\mathrm{p} K_{\mathrm{a}}$ and is used in the field of biomaterials, including glucose sensers, ${ }^{30-33}$ polymeric micelles ${ }^{34}$ and hydrogel preparation. ${ }^{35}$ During AC electrophoresis, the negatively charged poly(MAPBA-co-DMAAm) oscillated and reacted with diols at the PVA gel interface, and then the PVA gels adhered to each other (Figure 4b). Therefore, when AC electrophoresis was used, we were able to adhere the like-charged and the non-ionic gels by 
<smiles>[Y]C1CC(CC(C)(C)C)OC(CCCC2OC(C(C)(C)C)CC(C([Y])(C)C(O)CCC)O2)O1</smiles>

PVA gel<smiles>[Y]C(C)C(CC(C)(C)C([Y])(C)C)C(=O)N(C)C</smiles>

PBA copolymer b

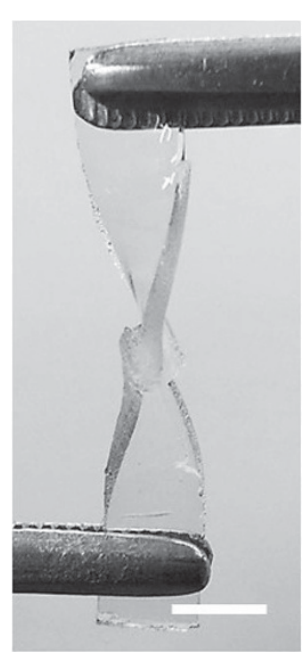

Figure 4 (a) The chemical structures of the poly(vinyl alcohol) (PVA) gel and the phenylboronic acid (PBA) copolymer. (b) A macroscopic image of adhered PVA/PVA hydrogels utilizing the PBA copolymer as a binder. The PVA hydrogels were stained with a green dye. The scale bar represents $5 \mathrm{~mm}$. Reproduced from Asoh et al. ${ }^{27}$ from the Royal Society of Chemistry. A full color version of this figure is available at Polymer Journal online.

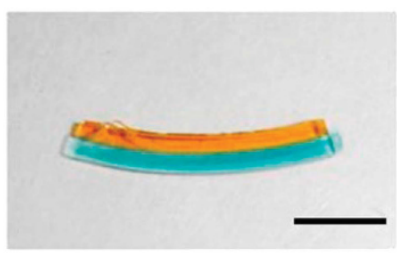

b

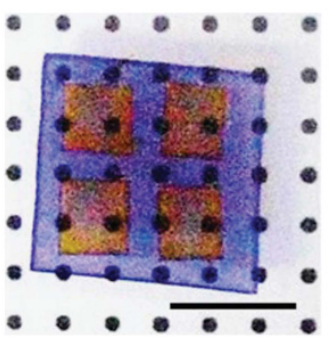

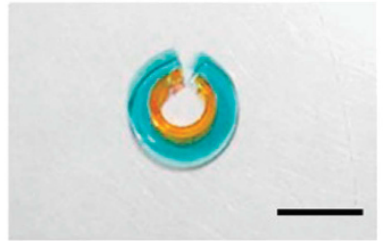

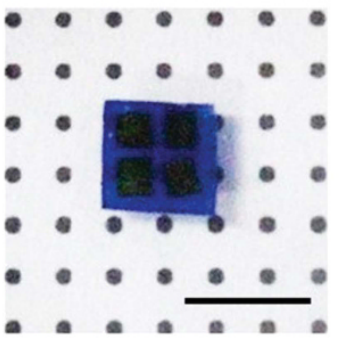

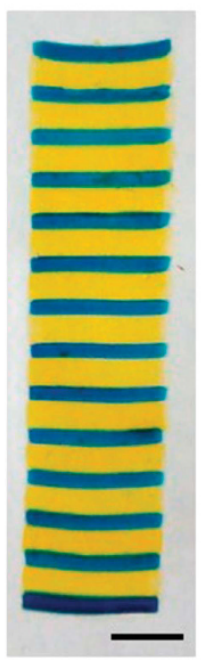

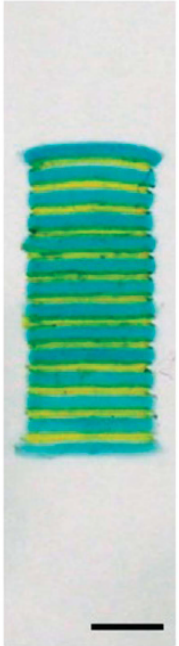

Figure 5 Images of (a) PNIPAAm/poly(acrylamide) (PAAm) bi-layered hydrogels, (b) PNIPAAm gel-array and (c) (PAAm/PNIPAAm) 13 PAAm multilayer hydrogels. The left and right images indicate the 20 and $40{ }^{\circ} \mathrm{C}$ in water, respectively. All scale bars are $5 \mathrm{~mm}$. Reproduced from Asoh et al..24,28 from the Royal Society of Chemistry.

exploiting the oscillation and stepwise interaction of water-soluble binders at the hydrogel interface.

\section{FREE-FORM FABRICATION BY ADHESION OF HYDROGELS}

The adhesion of hydrogels is a useful tool for the preparation of hydrogel constructs. Therefore, stimuli-responsive bending gels, a hydrogel array and a uniaxially moved soft actuator were prepared by the adhesion of thermoresponsive poly( $N$-isopropylacrylmide) gels (Figure 5). ${ }^{24,28}$ However, there is a limit to preparing highly organized 3D architectures through the adhesion of bulk hydrogels with milliand/or centimeter scales. Therefore, we attempted to apply electrophoretic adhesion of hydrogels to the rapid fabrication of hydrogel constructs. $^{29}$ We used PVA microgels with cationic poly(diallyldimethylammonium chloride) or anionic sodium poly(styrene sulfonate) (PSS) as the building blocks. Because the average diameter of the microgels was ca. $160 \mu \mathrm{m}$, the hydrogel constructs with milliand/or centimeter scales were fabricated by the adhesion of microgels with three-dimensionally. When a small amount of water was added to the same amount of cationic and anionic microgels, a pasty material was formed. Interestingly, the microgel paste gelled and then formed hydrogel constructs after the application of an electric field. The hydrogel constructs had elasticity and were sufficiently durable to be handled with forceps (Figure 6a). The leakage of microgels from these constructs was not observed in water after the removal of the electric field. As shown in Figures $3 \mathrm{~d}$ and $6 \mathrm{~b}$ microgel networks were formed through the adhesion of cationic and anionic microgels. The reconstruction of hydrogel constructs was possible by grinding and re-adhesion (Figure 6c). By grinding to break the adhesive interfaces, 
a

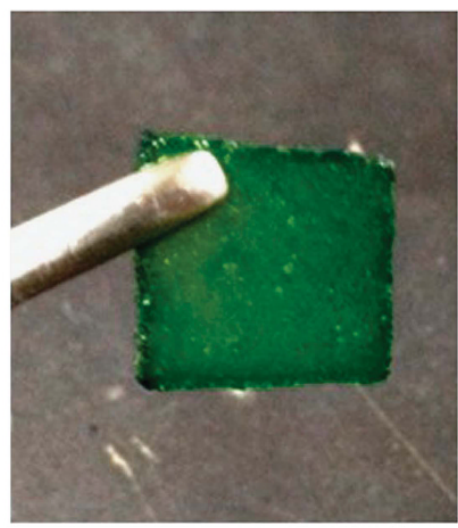

b

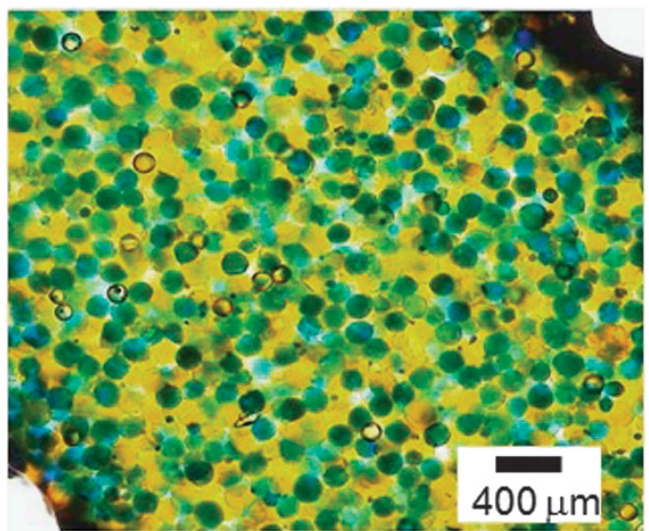

C
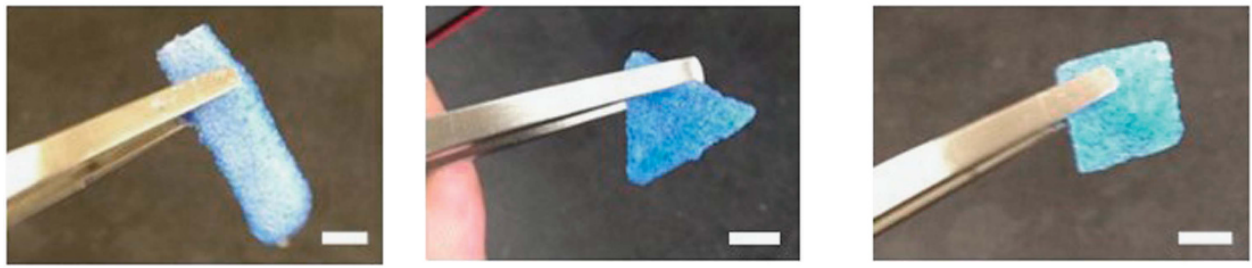

$\left.i\right|^{i i}$

iii
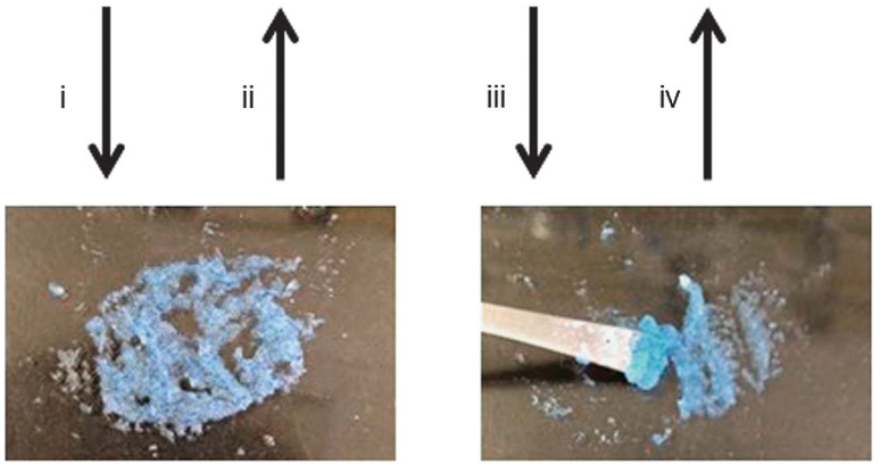

Figure 6 (a) Macroscopic and (b) microscopic images of hydrogel constructs prepared by the adhesion of cationic and anionic microgels. The cationic and anionic microgels were stained with orange and blue dyes. The size of the square-shaped hydrogel constructs was $10 \times 10 \mathrm{~mm}$, and the thickness is $1 \mathrm{~mm}$. (c) The images of reconstruction of hydrogel constructs. The anionic microgels were stained by a blue dye. All of the scale bars are $5 \mathrm{~mm}$. Reproduced from Asoh et al. ${ }^{29}$ from the Royal Society of Chemistry.

a

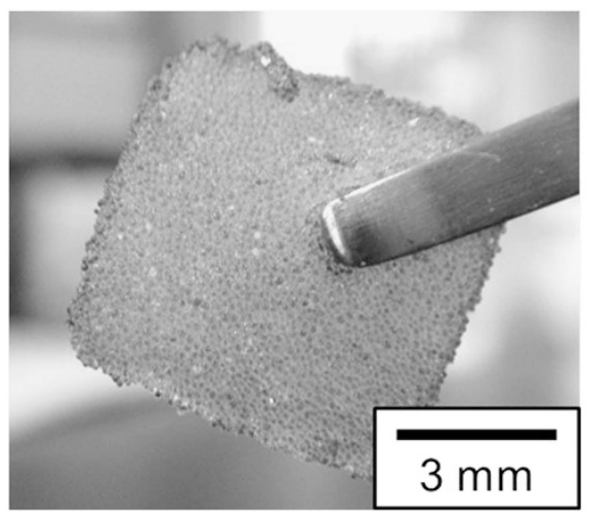

b

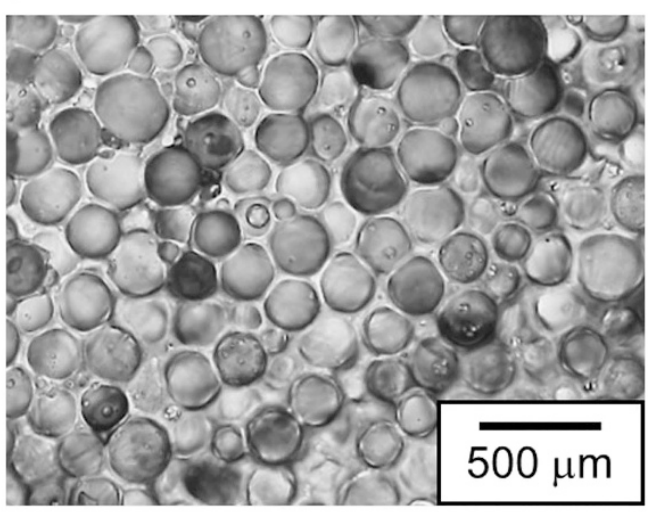

Figure 7 (a) Macroscopic and (b) microscopic images of hydrogel constructs prepared by adhesion of dextran sulfate (DS) microgels. The DS microgels were stained with a blue dye. Reproduced from Asoh et al. ${ }^{26}$ from Elsevier. A full color version of this figure is available at Polymer Journal online. 
we were able to change strip-shaped hydrogel constructs back into microgel paste (Figure $6 \mathrm{c}, \mathrm{i}$ ). The broken constructs were changed into triangle-shaped hydrogel constructs by the molding and electrophoretic re-adhesion of the microgel paste (Figure 6c, ii). Thereafter, square-shaped hydrogel constructs were prepared in a similar way (Figure $6 \mathrm{c}$, iii and iv). Biodegradable 3D microgel networks ${ }^{26}$ were also prepared by the adhesion of anionic DS microgels using cationic TMC as binders. A microgel paste composed of DS microgels and TMC was gelled by applying AC electric fields along the $x, y$ and $z$ axes (Figure 7a). As shown in Figure 7b, the microgel networks were formed through the adhesion of the DS microgels (Figure 7b). These results indicate that electrophoretic microgel adhesion is a powerful method for the free-form fabrication of hydrogel constructs.

\section{CONCLUSIONS AND OUTLOOK}

This paper summarizes the electrophoretic adhesion of hydrogels for use in the fabrication of functional 3D architectures that have recently been developed by our research group. ${ }^{24-29}$ Oppositely charged and like-charged gels were adhered to each other by DC and AC electrophoretic adhesion. The non-ionic PVA gel was also adhered by a combination of AC electrophoretic adhesion and PBA copolymer as a binder. To apply this adhesive method to build 3D materials, rapid and free-form fabrication of hydrogel constructs was achieved through the electrophoretic adhesion of microgels. However, the interface structure of the adhered gels is mostly unknown. Most recently, we have found wrinkled structures around the adhered gel interface, and the presence of the wrinkled structure may strengthen the hydrogel adhesion. ${ }^{36}$ The development of a novel adhesion strategy and an understanding of the interface of soft materials will be useful in the fabrication of novel 3D hydrogel architectures.

\section{CONFLICT OF INTEREST}

The authors declare no conflict of interest.

\section{ACKNOWLEDGEMENTS}

This study was supported by a Grant-in-Aid for Young Scientists (B) (No. 22700497) and a Grant-in-Aid for Challenging Exploratory Research (No. 26560227) from the Japan Society for the Promotion of Science, the Foundation for the Promotion of Ion Engineering and the Sasakawa Scientific Research Grant from The Japan Science Society. I am deeply indebted to Professor Akihiko Kikuchi (Tokyo University of Science) for his continuous encouragement and discussion throughout this work. I thank all of my students for their great contributions to this study. I also thank Professors Yasuyuki Tsuboi, Tetsuro Shinada, Yutaka Amao and Tatsuya Shoji, (Osaka City University) for discussions.

1 Lee, K. Y. \& Mooney, D. J. Hydrogels for tissue engineering. Chem. Rev. 101, 1869-1880 (2001)

2 Vermonden, T., Censi, R. \& Hennink, W. E. Hydrogels for protein delivery. Chem. Rev. 112, 2853-2888 (2012).

3 Osada, Y., Okuzaki, H. \& Hori, H. A polymer gel with electrically driven motility. Nature 355, 242-244 (1992).

$4 \mathrm{Hu}, \mathrm{Z}$., Zhang, X. \& Li, Y. Synthesis and application of modulated polymer gels. Science 269, 525-527 (1995).

5 Maeda, S., Hara, Y., Sakai, T., Yoshida, R. \& Hashimoto, S. Self-walking gel. Adv. Mater. 19, 3480-3484 (2007).

6 Asoh, T., Matsusaki, M., Kaneko, T. \& Akashi, M. Fabrication of temperature-responsive bending hydrogels with a nanostructured gradient. Adv. Mater. 20, 2080-2083 (2008).

7 Matsusaki, M., Kadowaki, K., Nakahara, Y. \& Akashi, M. Fabrication of cellular multilayers with nanometer-sized extracellular matrix films. Angew. Chem. Int. Ed. 46, 4689-4692 (2007)
8 Nishiguchi, A., Yoshida, H., Matsusaki, M. \& Akashi, M. Rapid construction of threedimensional multilayered tissues with endothelial tube networks by the cellaccumulation technique. Adv. Mater. 23, 3506-3510 (2011).

9 Matsunaga, Y. T., Morimoto, Y. \& Takeuchi, S. Molding cell beads for rapid construction of macroscopic 3D tissue architecture. Adv. Mater. 23, H90-H94 (2011).

10 Derby, B. Printing and prototyping of tissues and scaffolds. Science 338, 921-926 (2012).

11 Hinton, T. J., Jallerat, Q., Palchesko, R. N., Park, J. H., Grodzicki, M. S., Sue, H. J., Ramadan, M. H., Hudson, A.R. \& Feinberg, A. W. Three-dimensional printing of complex biological structures by freeform reversible embedding of suspended hydrogels. Sci. Adv. 1, e1500758 (2015)

$12 \mathrm{Wu}$, W., DeConinck, A. \& Lewis, J. A. Omnidirectional printing of 3D microvascular networks. Adv. Mater. 23, H178-H183 (2011).

13 Kolesky, D., Truby, R. L., Gladman, A. S., Busbee, T. A., Homan, K. A. \& Lewis, J. A. 3D bioprinting of vascularized, heterogeneous cell-laden tissue constructs. Adv. Mater. 26, 3124-3130 (2014).

14 Holten-Andersen, N., Harrington, M. J., Birkedal, H., Lee, B. P., Messersmith, P. B., Lee, K. Y. C. \& Waite, J. H. pH-induced metal-ligand cross-links inspired by mussel yield self-healing polymer networks with near-covalent elastic moduli. Proc. Natl Acad. Sci. USA 108, 2651-2655 (2011).

15 Tamagawa, H., Nagato, F., Umemoto, S., Okui, N., Popovic, S. \& Taya, M. The influence of salt-linkages on the adhesion and hardness variance of hydrogels. Bull. Chem. Soc. Jpn 75, 383-388 (2002).

16 Tamagawa, H. \& Takahashi, Y. Adhesion force behavior between two gels attached with an electrolytic polymer liquid. Mater. Chem. Phys. 107, 164-170 (2008).

17 Abe, H., Hara, Y., Maeda, S. \& Hashimoto, S. Surface modification method for adhesion of gels. Chem. Lett. 43, 243-245 (2014).

18 Sun, T. L., Kurokawa, T., Kuroda, S., Ihsan, A. B., Akasaki, T., Sato, K., Haque, M. A., Nakajima, T. \& Gong, J. P. Physical hydrogels composed of polyampholytes demonstrate high toughness and viscoelasticity. Nat. Mater. 12, 932-937 (2013)

19 Harada, A., Kobayashi, R., Takashima, Y., Hashidzume, A. \& Yamaguchi, H. Macroscopic self-assembly through molecular recognition. Nat. Chem. 3, 34-37 (2011).

20 Haraguchi, K., Uyama, K. \& Tanimoto, H. Self-healing in nanocomposite hydrogels. Macromol. Rapid Commun. 32, 1253-1258 (2011).

21 Wang, Q., Mynar, J. L., Yoshida, M., Lee, E., Lee, M., Okuro, K., Kinbara, K. \& Aida, T. High-water-content mouldable hydrogels by mixing clay and a dendritic molecular binder. Nature 463, 339-343 (2010).

22 Rosel, S., Prevoteau, A., Elzie're, P., Hourdet, D., Marcellan, A. \& Leibler, L. Nanoparticle solutions as adhesives for gels and biological tissues. Nature 505, 382-385 (2014).

23 Meddahi-Pelle', A., Legrand, A., Marcellan, A., Louedec, L., Letourneur, D. \& Leibler, L. Organ repair, hemostasis, and in vivo bonding of medical devices by aqueous solutions of nanoparticles. Angew. Chem. Int. Ed. 53, 6369-6373 (2014).

24 Asoh, T. \& Kikuchi, A. Electrophoretic adhesion of stimuli-responsive hydrogels. Chem. Commun. 46, 7793-7795 (2010).

25 Asoh, T., Kawai, W. \& Kikuchi, A. Electrophoretic adhesion of biodegradable hydrogels through the intermediary of oppositely charged polyelectrolytes. Soft Matter 8, 1923-1927 (2012)

26 Asoh, T., Kawai, W. \& Kikuchi, A. Alternating-current electrophoretic adhesion of biodegradable hydrogel utilizing intermediate polymers. Colloids Surf. B 123, 742-746 (2014).

27 Asoh, T., Takaishi, K. \& Kikuchi, A. Adhesion of poly(vinyl alcohol) hydrogels by electrophoretic manipulation of phenylboronic acid copolymer. J. Mater. Chem. B $\mathbf{3}$, 6740-6745 (2015)

28 Asoh, T., Kawamura, E. \& Kikuchi, A. Stabilization of electrophoretically adhered gelinterfaces to construct multi-layered hydrogels. RSC Adv. 3, 7947-7952 (2013).

29 Asoh, T. \& Kikuchi, A. Rapid fabrication of reconstructible hydrogels by electrophoretic microbead adhesion. Chem. Commun. 48, 10019-10021 (2012).

30 Kikuchi, A., Suzuki, K., Okabayashi, O., Hoshino, H., Kataoka, K., Sakurai, Y. \& Okano, T. Glucose-sensing electrode coated with polymer complex gel containing phenylboronic acid. Anal. Chem. 68, 823-828 (1996).

31 Kataoka, K., Miyazki, H., Bunya, M., Okano, T. \& Sakurai, Y. Totally synthetic polymer gels responding to external glucose concentration: their preparation and application to on-off regulation of insulin release. J. Am. Chem. Soc. 120, 12694-12695 (1998).

32 Alexeev, V. L., Sharma, A. C., Goponenko, A. V., Das, S., Lednev, I. K., Wilcox, C. S., Finegold, D. N. \& Asher, S. A. High ionic strength glucose-sensing photonic crystal. Anal. Chem. 75, 2316-2323 (2003).

33 Matsumoto, A., Ishii, T., Nishida, J., Matsumoto, H., Kataoka, K. \& Miyahara, Y. A synthetic approach toward a self-regulated insulin delivery system. Angew. Chem. Int. Ed. 51, 2124-2128 (2012).

34 Naito, M., Ishii, T., Matsumoto, A., Miyata, K., Miyahara, Y. \& Kataoka, K. A phenylboronate-functionalized polyion complex micelle for ATP-triggered release of siRnA. Angew. Chem. Int. Ed. 51, 10751-10755 (2012).

35 Konno, T. \& Ishihara, K. Temporal and spatially controllable cell encapsulation using a water-soluble phospholipid polymer with phenylboronic acid moiety. Biomaterials $\mathbf{2 8}$, 1770-1777 (2007)

36 Kato, M., Tsuboi, Y, Kikuchi, A. \& Asoh, T. Hydrogel adhesion with wrinkle formation by spatial control of polymer networks. J. Phys. Chem. B 120, 5042-5046 (2016). 


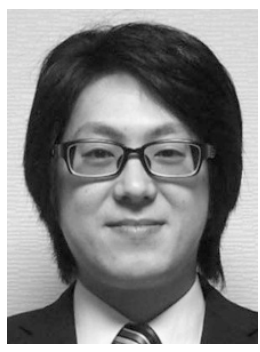

Dr Taka-Aki Asoh was born in Tanegashima (Kagoshima Prefecture), Japan in 1981. He received his BS degree from Kagoshima University in 2003, and his $\mathrm{PhD}$ degree of engineering from Osaka University in 2008, under the supervision of Professor Mitsuru Akashi. He was a postdoctoral researcher of Japan Society for the Promotion of Science (JSPS) in Osaka University under the supervision of Prof. Mitsuru Akashi (2008-2009). He joined the research group of Professor Akihiko Kikuchi in 2009 as an assistant professor in Tokyo University of Science. Since 2014, he has been a tenure-track lecturer of Advanced Research Institute for Natural Science and Technology, Osaka City University. He was a recipient of the Award for Encouragement of Research in Polymer Science (2015), from The Society of Polymer Science, Japan, Japanese and Korean Biomaterials Societies Young Scientist Exchange Program Award (2014), from Japanese Society for Biomaterials and several other awards. His current research interests include adhesion of hydrogels, stimuliresponsive polymers and gels as biomaterials, and design of degradable polymers and gels. 